Research Article

\title{
Heteroclinic Cycles Imply Chaos and Are Structurally Stable
}

\author{
Xiaoying Wu \\ School of Financial Mathematics and Statistics, Guangdong University of Finance, Guangzhou 510521, China \\ Correspondence should be addressed to Xiaoying Wu; wxyingyy@163.com
}

Received 19 December 2020; Revised 20 April 2021; Accepted 11 May 2021; Published 29 May 2021

Academic Editor: Abdul Qadeer Khan

Copyright $@ 2021$ Xiaoying Wu. This is an open access article distributed under the Creative Commons Attribution License, which permits unrestricted use, distribution, and reproduction in any medium, provided the original work is properly cited.

This paper is concerned with the chaos of discrete dynamical systems. A new concept of heteroclinic cycles connecting expanding periodic points is raised, and by a novel method, we prove an invariant subsystem is topologically conjugate to the one-side symbolic system. Thus, heteroclinic cycles imply chaos in the sense of Devaney. In addition, if a continuous differential map $h$ has heteroclinic cycles in $\mathbb{R}^{n}$, then $g$ has heteroclinic cycles with $\|h-g\|_{C^{1}}$ being sufficiently small. The results demonstrate $C^{1}$ structural stability of heteroclinic cycles. In the end, two examples are given to illustrate our theoretical results and applications.

\section{Introduction}

Since Li and Yorke first introduced the term "chaos" in 1975 [1], chaotic dynamics have been observed in various fields [2-10]. When chaotic theory was in its initial stage, Marotto generalized the results of $\mathrm{Li}$ and Yorke in interval mapping to multidimensional discrete systems and proved that a snapback repeller implies chaos [11]. In [2], Blanco demonstrated that $f: \mathbb{R}^{n} \longrightarrow \mathbb{R}^{n}$ has positive topological entropy if $f$ has snapback repellers. In 1989, Devaney gave an explicit chaotic definition which is called Devaney's chaos in [12]. In order to understand the relationship between various kinds of chaos, Huang and Ye obtained that Devaney's chaos implies Li-Yorke chaos in [13]. Based on the research of Marotto, Shi and Chen raised the concept of snapback repellers in Banach spaces and complete metric spaces $[14,15]$. As is well known, some homoclinic and heteroclinic cycles imply chaos in dynamical systems $[6,16]$. Lin and Chen introduced the new chaotic criteria of heteroclinic repellers in $\mathbb{R}^{n}$ [17]. Recently, Li et al. generalized the definition of heteroclinic repellers to infinite dimensional dynamical systems [18].

From the view of application, we naturally ask whether a chaotic dynamical system still has chaotic behaviors under small perturbations. In [19], Marotto showed that the systems with snapback repellers under delayed perturbations still have chaotic behaviors. In [20], Li et al. showed the $C^{1}$ structural stability of snapback repellers in $\mathbb{R}^{n}$. Further, Chen et al. studied the structure stability of snapback repellers in Banach space [21]. Chen and Li provided a sufficient condition of a high-dimensional difference equation having symbolic embedding for enough small $C^{1}$ perturbations [22]. In 2020, Chen and $\mathrm{Wu}$ et al. showed that the system with heteroclinic repellers has the structural stability in $[4,16]$.

In this paper, we consider the following discrete dynamical systems:

$$
x(n+1)=f(x(n)), \quad n=1,2, \ldots,
$$

where $f$ is a continuous map from $\mathbb{R}^{n}$ into itself. Based on the concept of heteroclinic repellers in discrete dynamical systems, we introduce a new concept of heteroclinic cycles connecting expanding periodic points in $\mathbb{R}^{n}$; by a novel method, we can construct shift invariant sets and prove that heteroclinic cycles imply chaos in the sense of Devaney. It is very important to choose an appropriate recurrent time set in the proof of the theorem. In particular, it is easier to find the conditions of heteroclinic cycles than that of snap back repellers for the system in [3]. The following theorems are the main results.

Theorem 1. Let $h$ be a continuous differential map from $\mathbb{R}^{n}$ into itself. If $h$ has heteroclinic cycles connecting expanding periodic points, then $h$ is chaotic in the sense of Devaney. 
Theorem 2. Let $g$, $h$ be continuous differential maps from $\mathbb{R}^{n}$ into itself. If $h$ has heteroclinic cycles connecting expanding periodic points and $\|g-h\|_{C^{1}}$ is sufficiently small, where $\|g-h\|_{C^{1}}=\max \left\{\sup _{x \in X}\|g(x)-h(x)\|, \sup _{x \in} X \| D g\right.$ $(x)-D h(x) \|\}, g$ also has heteroclinic cycles connecting expanding periodic points.

This paper is organized as follows: in Section 1, the relevant results of the previous studies are introduced. In Section 2, some definitions and necessary lemmas are given. A new concept of heteroclinic cycles in the sequel is raised. In Section 3, the main conclusions are drawn. At the end, some examples are presented to illustrate the main results and applications in Section 4.

\section{Definitions and Lemmas}

In this section, some definitions and lemmas will be introduced.

Definition 1. Let $f: \mathbb{R}^{n} \longrightarrow \mathbb{R}^{n}$ be a continuous differentiable map. $f$ is expanding at a point $x_{0}$ if the derivative operator $D f\left(x_{0}\right)$ is invertible and the norm of each of its eigenvalues is larger than 1. $f$ is expanding in a nonempty subset $U$ of $\mathbb{R}^{n}$ if there exists positive constant $\lambda>1$ such that, for any $x, y \in U,\|f(x)-f(y)\| \geq \lambda\|x-y\|$.

It is well known that if $f$ is differentiable, then $f$ is expanding at a point $x_{0}$ which is equivalent to the fact that there exist a norm $\|\cdot\|$ in $\mathbb{R}^{n}$ and a constant $\lambda>1$ such that $\forall x \in \mathbb{R}^{n},\left\|D f\left(x_{0}\right) x\right\| \geq \lambda\|x\|$ [3]. The following heteroclinic repellers in $\mathbb{R}^{n}$ were presented by Lin and Chen in [17]. where $t(i) \equiv i(\bmod k)$.

Definition 2 (see [17]). Let $f: \mathbb{R}^{n} \longrightarrow \mathbb{R}^{n}$ be a continuous differentiable map and the integer $k \geq 2$ be fixed. The fixed points $x_{1}, x_{2}, \ldots, x_{k}$ of $f$ are called heteroclinic repellers if for any integer $1 \leq i \leq k$, there are

(1) $f$ is expanding at the point $x_{i}$

(2) $f$ has a forward orbit $\Gamma_{i}=\left\{z_{i}(-n)\right\}_{n \geq 0}$ of $x_{t(i)+1}$ such that $\forall n \in \mathbb{N} \cap[1,+\infty), f\left(z_{i}(-n)\right)=z_{i}(-n+1)$ and $z_{i}(0)=x_{t(i)+1}, \lim _{n \longrightarrow+\infty} z_{i}(-n)=x_{i}$

(3) $f$ is continuously differentiable in a neighborhood of any point $z$ on $\Gamma_{i}$ and $D f(z)$ is invertible,

Based on Definition 2, we introduce the following new concept.

Definition 3. Let $f$ be a continuous differentiable map from $\mathbb{R}^{n}$ into itself and $k \geq 1$ be fixed integer. Let $f$ have $k$ different periodic points $x_{1}, x_{2}, \ldots, x_{k}$ with periods $n_{1}, n_{2}, \ldots, n_{k}$, respectively. For any $1 \leq m \leq n_{i}$ and $i \neq j, f^{m}\left(x_{i}\right) \neq x_{j}$. For any $1 \leq i \leq k$, suppose that

(1) $f$ is expanding at every point $x$ which is in the periodic orbits of $x_{i}$.

(2) $f$ has a forward orbit $\Gamma_{i}=\left\{z_{i}(-n)\right\}_{n \geq 0}$ of $x_{t(i)+1}$ connecting periodic points $x_{i}$ and $x_{t(i)+1}$. That is, it

$$
\begin{aligned}
& \text { satisfies that } \forall n \in \mathbb{N} \cap[1,+\infty), \\
& f\left(z_{i}(-n)\right)=z_{i}(-n+1), z_{i}(0)=x_{t(i)+1} \text { and there } \\
& \text { exists a positive integer } m_{i} \text { such that } \\
& \lim _{n \longrightarrow+\infty} z_{i}\left(-n n_{i}-m_{i}\right)=x_{i} .
\end{aligned}
$$

(3) For any point $z$ on $\Gamma_{i}$, the linear operator $D f(z): \mathbb{R}^{n} \longrightarrow \mathbb{R}^{n}$ is invertible.

Then, the set $\cup_{i=1}^{k} \Gamma_{i}$ is called a heteroclinic cycle connecting expanding periodic points $x_{1}, x_{2}, \ldots, x_{k}$.

Remark 1. By Definition 3, for any point $z$ in heteroclinic cycles connecting expanding periodic points $x_{1}, x_{2}, \ldots, x_{k}$, $D h(z)$ is invertible and the norm of each of its eigenvalues is larger than 1 . Thus, $x_{1}, x_{2}, \ldots, x_{k}$ are heteroclinic repellers when every periodic point is fixed point in Definition 3 and $k \geq 2$. Example 1 shows that heteroclinic cycles connecting expanding periodic points are different from heteroclinic repellers.

To prove the $C^{1}$ structural stability of heteroclinic cycles connecting expanding periodic points, we need the following implicit function theorem with parametric variables and continuous dependence theorem of inverse mapping.

Lemma 1 (see [21]). Let $(X,\|\cdot\|)$ and $(Y,\|\cdot\|)$ be Banach spaces, $(\Lambda, d)$ be a metric space, and $U$ be an open set of $\Lambda \times X$. Suppose that $F: U \longrightarrow Y$ is a continuous differentiable map and there exists a point $\left(\lambda_{0}, x_{0}\right) \in U$ satisfying the following conditions:

(1) $D F_{x}(\lambda, x)$, the Fr e chet partial derivative of $F(\lambda, x)$ with respect to $x$, is continuous with respect to $x$ in some neighborhood of $\left(\lambda_{0}, x_{0}\right)$

(2) $D F_{x}\left(\lambda_{0}, x_{0}\right): X \longrightarrow Y$ is an invertible linear operator

(3) $F\left(\lambda_{0}, x_{0}\right)=0$,

Then, there exist open ball $B_{r_{1}}\left(x_{0}\right)=\left\{x \mid\left\|x-x_{0}\right\|<r_{1}\right\}$ and $B_{\delta}\left(\lambda_{0}\right)=\left\{\lambda \mid d\left(\lambda, \lambda_{0}\right)<\delta\right\}$, where $r_{1}>0, \delta>0$, such that for any $\lambda \in B_{\delta}\left(\lambda_{0}\right), F(\lambda, x)=0$, the unique continuous solution $x=h(\lambda) \in B_{r_{1}}\left(x_{0}\right)$ exists and $x_{0}=h\left(\lambda_{0}\right)$ is satisfied.

Lemma 2 (see [21]). Let $(Y,\|\cdot\|)$ and $(Z,\|\cdot\|)$ be two Banach spaces and $V_{0}=B_{r}\left(y_{0}\right)$ be $r$-open ball neighborhood of $y_{0}$. Assume that $h$ is a $C^{p}$ map from $V_{0}$ into $Z$ such that $h\left(y_{0}\right)=z_{0}(p \geq 1)$ and $D h\left(y_{0}\right)$ is an invertible linear operator from $Y$ into $Z$. Then, there are constants $r_{0}>0, \delta_{0}>0, \lambda_{0}>0$ and a map from $\mathscr{V}\left(h, \lambda_{0}\right) \times B_{\delta_{0}}\left(z_{0}\right)$ into $B_{r_{0}}\left(y_{0}\right)$ satisfying the following conditions:

(1) For any $\varphi \in \mathscr{V}\left(h, \lambda_{0}\right), \varphi$ is one to one on $B_{r_{0}}\left(y_{0}\right)$, and $D \varphi(y)$ is invertible for every $y \in B_{r_{0}}\left(y_{0}\right)$

(2) For any $\varphi \in \mathscr{V}\left(h, \lambda_{0}\right)$ and $z \in B_{\delta_{0}}\left(z_{0}\right)$, there is a unique $f(\varphi, z) \in B_{r_{0}}\left(y_{0}\right)$ satisfying $\varphi(f(\varphi, z))=z$

(3) The map $f$ is a continuous map from $\mathscr{V}\left(h, \lambda_{0}\right) \times$ $B_{\delta_{0}}\left(z_{0}\right)$ into $B_{r_{0}}\left(y_{0}\right)$

(4) For every $\varphi \in \mathscr{V}\left(h, \lambda_{0}\right), f(\varphi, \cdot): B_{\delta_{0}}\left(z_{0}\right) \longrightarrow B_{r_{0}}\left(y_{0}\right)$ is a p-order continuous differentiable map, and $D_{z} f(\varphi, z)=(D \varphi(f(\varphi, z)))^{-1}$, 
where

$$
\mathscr{V}\left(h, \lambda_{0}\right)=\left\{\varphi \mid\|\varphi-h\|_{C^{1}}<\lambda_{0}, \quad \varphi \in C^{p}\left(V_{0}, Z\right)\right\},
$$

denotes the $\lambda_{0}-C^{1}$-open ball neighborhood of $h$ and $C^{p}\left(V_{0}, Z\right)$ is a p-order continuous differentiable map set.

Lemma 3. Let $h$ be a continuous differentiable map from $\mathbb{R}^{n}$ into itself. Let $h$ have $k \geq 2$ periodic points $x_{1}, x_{2}, \ldots, x_{k}$ with periods $n_{1}, n_{2}, \ldots, n_{k}$, respectively. If $h$ is expanding at every point $x$ which is in the periodic orbit of $x_{i}$, then for all $1 \leq i \leq k$, there exist positive constants $r_{i}$ and $\lambda>0$ such that $\varphi^{n_{i}}$ is homeomorphism on closed ball $\bar{B}_{r_{i 0}}\left(x_{i}\right)$ for every $\varphi \in \mathscr{V}(h, \lambda)$ and $r_{i 0}<r_{i}, \varphi^{n_{i}}$ is expanding on $\vec{B}_{r_{i 0}}\left(x_{i}\right)$ and

$$
\begin{aligned}
& B_{r_{i 0}}\left(x_{i}\right) \subset \varphi^{n_{i}}\left(B_{r_{i 0}}\left(x_{i}\right)\right), \\
& \bar{B}_{r_{i 0}}\left(x_{i}\right) \subset \varphi^{n_{i}}\left(\bar{B}_{r_{i 0}}\left(x_{i}\right)\right) .
\end{aligned}
$$

Proof. For any $1 \leq i \leq k$, because $h$ is expanding at every point $x$ which is in the periodic orbit of $x_{i}$, by the continuity of $h$, there exists some closed neighborhood $\bar{B}_{r_{i}}\left(x_{i}\right)$ of $x_{i}$ such that $h$ is expanding in $h^{m}\left(\bar{B}_{r_{i}}\left(x_{i}\right)\right)$ for $0 \leq m \leq n_{i}-1$. Therefore, there exists a positive constant $\lambda_{i}>1$ such that, for all $0 \leq m \leq n_{i}-1$ and for any $x, y \in h^{m}\left(\bar{B}_{r_{i}}\left(x_{i}\right)\right)$, there is

$$
\|h(x)-h(y)\| \geq \lambda_{i}\|x-y\| .
$$

Let $\lambda_{i 1} \in\left(1, \lambda_{i}\right)$. Thus, for every $\varphi \in \mathscr{V}\left(h, \lambda_{i}-\lambda_{i 1}\right)$ and $x, y \in B_{r_{i}}\left(x_{i}\right)$, there is

$$
\begin{aligned}
\left\|\varphi^{n_{i}}(x)-\varphi^{n_{i}}(y)\right\| & \geq\left\|h\left(\varphi^{n_{i}-1}(x)\right)-h\left(\varphi^{n_{i}-1}(y)\right)\right\|-\left\|(\varphi-h)\left(\varphi^{n_{i}-1}(x)\right)-(\varphi-h)\left(\varphi^{n_{i}-1}(y)\right)\right\| \\
& >\left[\lambda_{i}-\left(\lambda_{i}-\lambda_{i 1}\right)\right]\left\|\varphi^{n_{i}-1}(x)-\varphi^{n_{i}-1}(y)\right\| \\
& =\lambda_{i 1}\left\|\varphi^{n_{i}-1}(x)-\varphi^{n_{i}-1}(y)\right\|>\lambda_{i 1}^{n_{i}}\|x-y\| .
\end{aligned}
$$

Because $h^{n_{i}}\left(x_{i}\right)=x_{i}$ and $D h^{n_{i}}\left(x_{i}\right): \mathbb{R}^{n} \longrightarrow \mathbb{R}^{n}$ is invertible, by Lemma 2, there are constants $0<\lambda_{i}^{\prime}<\lambda_{i}-\lambda_{i 1}, 0<r_{i 0}<r_{i}$ such that for every $\varphi \in \mathscr{V}\left(h, \lambda_{i}^{\prime}\right)$, $\varphi^{n_{i}}: B_{r_{i 0}}\left(x_{i}\right) \longrightarrow \varphi^{n_{i}}\left(B_{r_{i 0}}\left(x_{i}\right)\right)$ is a differentiable homeomorphism. Therefore, for any $1 \leq m \leq n_{i}$, $\varphi: \varphi^{m-1}\left(B_{r_{i 0}}\left(x_{i}\right)\right) \longrightarrow \varphi^{m}\left(B_{r_{i 0}}\left(x_{i}\right)\right)$ is a differentiable homeomorphism and

$$
\left.\varphi^{m}\left(\bar{B}_{r_{i 0}}\left(x_{i}\right)\right) \supset \overline{\varphi^{m}\left(\bar{B}_{r_{i 0}}\left(x_{i}\right)\right)}, \varphi^{m}\left(\partial B_{r_{i 0}}\left(x_{i}\right)\right) \supset \partial \varphi^{m}\left(B_{r_{i 0}}\left(x_{i}\right)\right)\right),
$$

where $\partial B_{r_{i 0}}\left(x_{i}\right)$ denotes the boundary of the set $B_{r_{i 0}}\left(x_{i}\right)$ and $\overline{\varphi^{m}\left(B_{r_{i 0}}\left(x_{i}\right)\right)}$ is the closure of the set $\varphi^{m}\left(B_{r_{i 0}}\left(x_{i}\right)\right)$. Take $\lambda_{i 2} \in\left(\max \left\{\lambda_{i 1},\left(\left(\lambda_{i}+r_{i 0}\right) /\left(1+r_{i 0}\right)\right)\right\}, \lambda_{i}\right)$. For every $\varphi \in \mathscr{V}\left(h,\left(\left(\lambda_{i}-\lambda_{i 2}\right) / 2\right)\right), x \in \partial B_{r_{i 0}}\left(x_{i}\right)$, there is

$$
\begin{aligned}
\left\|\varphi(x)-\varphi\left(x_{i}\right)\right\| \geq & \left\|h(x)-h\left(x_{i}\right)\right\| \\
& -\left\|\varphi(x)-h(x)-\varphi\left(x_{i}\right)+h\left(x_{i}\right)\right\| \\
& >\lambda_{i 2} r_{i 0}-\left(\lambda_{i}-\lambda_{i 2}\right)>r_{i 0} .
\end{aligned}
$$

Hence,

$\varphi\left(B_{r_{i 0}}\left(x_{i}\right)\right) \supset B_{r_{i 0}}\left(\varphi\left(x_{i}\right)\right), \varphi\left(\bar{B}_{r_{i 0}}\left(x_{i}\right)\right) \supset \bar{B}_{r_{i 0}}\left(\varphi\left(x_{i}\right)\right)$. Inductively, for every $0 \leq m \leq n_{i}-1$, there are

$$
\begin{aligned}
& \varphi\left(B_{r_{i 0}}\left(\varphi^{m}\left(x_{i}\right)\right)\right) \supset B_{r_{i 0}}\left(\varphi^{m}\left(x_{i}\right)\right), \\
& \varphi\left(\bar{B}_{r_{i 0}}\left(\varphi^{m}\left(x_{i}\right)\right)\right) \supset \bar{B}_{r_{i 0}}\left(\varphi^{m}\left(x_{i}\right)\right) .
\end{aligned}
$$

Let $\lambda=\min \left\{\lambda_{i}^{\prime}, \lambda_{i}-\lambda_{i 2} \mid 1 \leq i \leq k\right\}$, and for every $\varphi \in \mathscr{V}(h, \lambda)$, there are

$$
\begin{aligned}
& B_{r_{i 0}}\left(x_{i}\right) \subset \varphi^{n_{i}}\left(B_{r_{i 0}}\left(x_{i}\right)\right), \\
& \bar{B}_{r_{i 0}}\left(x_{i}\right) \subset \varphi^{n_{i}}\left(\bar{B}_{r_{i 0}}\left(x_{i}\right)\right) .
\end{aligned}
$$

The proof of the lemma is done.

\section{Heteroclinic Cycles Connecting Expanding Periodic Points Imply Chaos and Are Structurally Stable}

In this section, we are going to consider that heteroclinic cycles imply Devaney's chaos and are structurally stable.

Theorem 3. Let $h$ be a continuous differential map from $\mathbb{R}^{n}$ into itself. If $h$ has heteroclinic cycles connecting expanding periodic points, $h$ is chaotic in the sense of Devaney.

Proof. Let $x_{1}, x_{2}, \ldots, x_{k}$ be $k \geq 1$ different periodic points with periods $n_{1}, n_{2}, \ldots, n_{i}$, respectively, and $h$ be expanding at $x_{1}, x_{2}, \ldots, x_{k}$, respectively. Let $\cup_{i=1}^{i=k} \Gamma_{i}=\left\{z_{i}(-n)\right\}_{n \geq 0}$ be heteroclinic cycles connecting periodic points $x_{1}, x_{2}, \ldots, x_{k}$.

For any integer $i \in[1, k]$ and any integer $j \geq 0$, by Definition 3, $D h\left(h^{j}\left(x_{i}\right)\right)$ is invertible and the norm of each of its eigenvalues is larger than 1. Therefore, there exist constants $r_{i}>0, \lambda_{i}>1$ such that, for any integer $m \in\left[0, n_{i}-\right.$ 1] and $x, y \in \bar{B}_{r_{i}}\left(h^{m}\left(x_{i}\right)\right),\|h(x)-h(y)\| \geq \lambda_{i}\|x-y\|$. There exists a positive integer $m_{i}$ such that $z_{i}\left(-m_{i}\right) \in B_{r_{i} / 2}\left(x_{i}\right)$ and $\lim _{n \longrightarrow+\infty} z_{i}\left(-n n_{i}-m_{i}\right)=x_{i}$. Without loss of generality, for any integer $i, j \in[1, k], m \in\left[1, m_{i}\right], l \in\left[1, m_{j}\right]$, let $\bar{B}_{r_{i}}\left(h^{m}\left(x_{i}\right)\right) \cap \bar{B}_{r_{j}}\left(h^{l}\left(x_{j}\right)\right)=\varnothing$, if $h^{m}\left(x_{i}\right) \neq h^{l}\left(x_{j}\right)$. Since $D h^{m_{i}}\left(z_{i}\left(-m_{i}\right)\right)$ is invertible, there exist positive constants 
$0<\widetilde{\delta}_{i}<\min \left\{(1 / 3)\left\|x_{i}-z_{i}\left(-m_{i}\right)\right\|,\left(r_{i} / 2\right)\right\}, \mu_{i}>0$ such that $h^{m_{i}}$ is a homeomorphism on $\bar{B}_{\delta_{i}}\left(z_{i}\left(-m_{i}\right)\right)$ and

$$
\begin{aligned}
\forall x, y & \in \bar{B}_{\widetilde{\delta}_{i}}\left(z_{i}\left(-m_{i}\right)\right), \\
\left|h^{m_{i}}(x)-h^{m_{i}}(y)\right| & \geq \mu_{i}^{m_{i}}|x-y| .
\end{aligned}
$$

By the continuity of $h$, there exists a closed ball $\bar{B}_{\delta_{1}}\left(x_{1}\right) \subset \bar{B}_{\delta_{1}}\left(x_{1}\right)$ such that

$$
\begin{aligned}
\left(\left.h\right|_{\bar{B}_{\delta_{k}}\left(z_{k}\left(-m_{k}\right)\right)}\right)^{-m_{k}}\left(\bar{B}_{\delta_{1}}\left(x_{1}\right)\right) & \subset \bar{B}_{\widetilde{\delta}_{k}}\left(z_{k}\left(-m_{k}\right)\right), \\
\bar{B}_{\delta_{1}}\left(x_{1}\right) & \cap \bar{B}_{\widetilde{\delta}_{1}}\left(z_{1}\left(-m_{1}\right)\right)=\varnothing,
\end{aligned}
$$

where $\left(\left.h\right|_{\bar{B}_{\delta_{k}}\left(z_{k}\left(-m_{k}\right)\right)}\right)^{-m_{k}}$ is the inverse of $h^{m_{k}}$ restricted to $\bar{B}_{\delta_{k}}\left(z_{k}\left(-m_{k}\right)\right)$. Similarly, for any integer $i \in[2, k]$, there exists $\bar{B}_{\delta_{i}}\left(x_{i}\right) \subset \bar{B}_{\delta_{i}}\left(x_{i}\right)$ such that

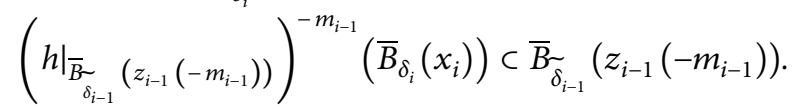

Next we prove there exist two nonempty bounded closed subsets $V_{0}$ and $V_{1}$ with $V_{0} \cap V_{1}=\varnothing$ such that, for a positive integer $n_{0}, h^{n_{0}}\left(V_{j}\right) \supset V_{0} \cup V_{1}, j=0,1$ and $h^{n_{0}}$ restricted to $V_{0}$ and $V_{1}$, respectively, is expanding. Because $h^{n_{i}}$ is expanding on $\bar{B}_{r_{i}}\left(x_{i}\right)$ and $x_{i}$ is a fixed point of $h^{n_{i}}$ for every $1 \leq i \leq k$, there are

(1) There exists a positive integer $\hat{n}_{1}$ such that $\left(\left.h^{n_{1}}\right|_{\bar{B}_{r_{1}}\left(x_{1}\right)}\right)^{-n_{1}}\left(\bar{B}_{r_{1} / 2}\left(x_{1}\right)\right) \subset \bar{B}_{\delta_{1}}\left(x_{1}\right)$.

(2) For every $2 \leq i \leq k$, there exists a positive integer $n_{i}$ such that

$$
\left(\left.h^{n_{i}}\right|_{\bar{B}_{r_{i}}\left(x_{i}\right)}\right)^{-\widehat{n}_{i}}\left(\bar{B}_{\delta_{i}}\left(z_{i}\left(-m_{i}\right)\right)\right) \subset \bar{B}_{\delta_{i}}\left(x_{i}\right) .
$$

Let $\lambda=\min \left\{\lambda_{i} \mid 1 \leq i \leq k\right\}$; then, there is $\lambda>1$. There exists a positive integer $n_{0}>\widehat{n}_{1} n_{1}+\sum_{i=1}^{k}\left(\widehat{n}_{i} n_{i}+m_{i}\right)$ such that $n_{0}-$ $\sum_{i=1}^{k}\left(\widehat{n}_{i} n_{i}+m_{i}\right) \equiv 0\left(\bmod n_{1}\right)$ and $\lambda^{n_{0}-\sum_{i=1}^{k} m_{i}} \prod_{i=1}^{k} \mu_{i}^{m_{i}}>1$. Let

$$
\begin{aligned}
& l \equiv n_{0}\left(\bmod n_{1}\right), \\
& V_{0}=\left(\left.h\right|_{\bar{B}_{r_{1}}\left(h^{n_{1}-l}\left(x_{1}\right)\right)}\right)^{-l}\left(\left(\left.h\right|_{\bar{B}_{r_{1}}\left(x_{1}\right)}\right)^{-\left(n_{0}-l\right)}\left(\bar{B}_{\left(r_{1} / 2\right)}\left(x_{1}\right)\right)\right), \\
& V_{k}=\left(\left.h\right|_{\bar{B}_{r_{k}}\left(x_{k}\right)}\right)^{-\widehat{n}_{k} n_{k}}\left(\left(\left.h\right|_{\bar{B}_{\delta_{k}}\left(z_{k}\left(-m_{k}\right)\right)}\right)^{-m_{k}}\left(\bar{B}_{\delta_{1}}\left(x_{1}\right)\right)\right) \subset \bar{B}_{\delta_{k}}\left(x_{k}\right) \text {, } \\
& V_{i}=\left(\left.h\right|_{\bar{B}_{r_{i}}\left(x_{i}\right)}\right)^{-\hat{n}_{i} n_{i}}\left(\left(\left.h\right|_{\bar{B}_{\delta_{i}}\left(z_{i}\left(-m_{i}\right)\right)}\right)^{-m_{i}}\left(V_{i+1}\right)\right), \quad \text { for all } 2 \leq i \leq k-1 \text {, } \\
& V_{1}=\left(\left.h\right|_{\bar{B}_{r_{i}}\left(x_{i}\right)}\right)^{-\left(n_{0}-\sum_{i=1}^{k}\left(\widehat{n}_{i} n_{i}+m_{i}\right)\right)}\left(\left(\left.h\right|_{\bar{B}_{\delta_{1}}\left(z_{1}\left(-m_{1}\right)\right)}\right)^{-m_{1}}\left(V_{2}\right)\right) \text {. }
\end{aligned}
$$

Then, $V_{0}$ and $V_{1}$ are two nonempty bounded closed subsets of $\mathbb{R}^{n}$ and

$$
\begin{aligned}
& V_{0} \subset \bar{B}_{r_{1}}\left(h^{n_{1}-l}\left(x_{1}\right)\right), \\
& V_{1} \subset \bar{B}_{\delta_{1}}\left(x_{1}\right) .
\end{aligned}
$$

Therefore, $V_{0} \cap V_{1}=\varnothing$. From definitions of $V_{0}$ and $V_{1}$, we have

$$
\begin{aligned}
& h^{n_{0}}\left(V_{0}\right)=\bar{B}_{r_{1} / 2}\left(x_{1}\right) \supset V_{0} \cup V_{1}, \\
& h^{n_{0}}\left(V_{1}\right) \supset \bar{B}_{r_{1} / 2}\left(x_{1}\right) \supset V_{0} \cup V_{1} .
\end{aligned}
$$

In addition, we have that

$$
\begin{aligned}
& \left\|h^{n_{0}}(x)-h^{n_{0}}(y)\right\|>\lambda^{n_{0}}\|x-y\|, \forall x, y \in V_{0}, \\
& \left\|h^{n_{0}}(x)-h^{n_{0}}(y)\right\|>\lambda^{n_{0}-} \sum_{i=1}^{k} m_{i} \prod_{i=1}^{k} \mu_{i}^{m_{i}}\|x-y\| \quad \forall x, y V_{1} .
\end{aligned}
$$

We claim that

$$
\begin{gathered}
\forall\left(i_{0}, i_{1}, \ldots, i_{n}, \ldots\right) \in \Sigma_{2}^{+}, \\
\#\left(\bigcap_{s=0}^{\infty} h^{-n_{0} s}\left(V_{i_{s}}\right)\right) \leq 1,
\end{gathered}
$$

where \#(.) denotes the cardinality of a set. 
Proceed the proof by contradiction. Suppose $x_{1} \neq x_{2}$ and $x_{1}, x_{2} \in \cap_{s=0}^{\infty} h^{-n_{0} s}\left(V_{i_{s}}\right)$. Let $\left(i_{0}, i_{1}, \ldots, i_{n}, \ldots\right) \in \Sigma_{2}^{+}$; then, there exists a subsequence $\left\{j_{n}\right\}_{n \in \mathbb{N}}$ of $\mathbb{N}$ such that for any $n \in \mathbb{N}, i_{j_{n}}=i_{j_{n+1}}$. Without loss of generality, for any $n \in \mathbb{N}, i_{j_{n}}=0$. By (17), we have

$$
\begin{aligned}
&\left\|h^{n_{0} j_{n}}\left(x_{1}\right)-h^{n_{0} j_{n}}\left(x_{2}\right)\right\| \geq \lambda^{n} \cdot\left(\lambda_{i=1}^{n_{0}-} \sum_{k m_{i}} \prod_{i=1}^{k} \mu_{i}^{m_{i}}\right)^{j_{n}-n} \\
& \cdot\left\|x_{1}-x_{2}\right\| \\
&>\lambda^{n}\left\|x_{1}-x_{2}\right\| \longrightarrow+\infty,
\end{aligned}
$$

which is contrary to the fact that $h^{n_{0} j_{n}}\left(x_{1}\right)$ and $h^{n_{0} j_{n}}\left(x_{2}\right)$ belong to the bounded set $V_{0}$. Therefore, there exist an invariant set $\Lambda_{0} \subset V_{0}$ and a positive integer $n_{0}$ such that $\left(\Lambda_{1}, h^{n_{0}}\right)$ is topologically conjugate to the one-side symbolic system $\left(\Sigma_{2}^{+}, \sigma\right)$. That is, $h^{n_{0}}$ is chaotic in the sense of Devaney and so is $h$.

From Theorem 3, we obtain the following corollary.

Corollary 1. Let $h$ be a continuous differentiable map from $\mathbb{R}^{n}$ into itself. If $h$ has heteroclinic repellers $x_{1}, x_{2}, \ldots, x_{k}$ with $k \geq 2$, then for every neighborhood $U_{i}$ of $x_{i}$, there exist an invariant subset $\Lambda_{i}$ of $U_{i}$ and a positive integer $n_{i}$ such that $\left(\Lambda_{i}, h^{n_{i}}\right)$ is topologically conjugate to the one-side symbolic system $\left(\Sigma_{2}^{+}, \sigma\right)$. Therefore, $h^{n_{i}}$ is chaotic in the sense of Devaney and so is $h$.

Theorem 4. Let $g$, $h$ be continuous differential maps from $\mathbb{R}^{n}$ into itself. If $h$ has heteroclinic cycles connecting expanding periodic points and $\|g-h\|_{C^{1}}$ is sufficiently small, then $g$ also has heteroclinic cycles connecting expanding periodic points.

Proof. Let $x_{1}, x_{2}, \ldots, x_{k}$ be $k \geq 1$ different periodic points with periods $n_{1}, n_{2}, \ldots, n_{i}$, respectively. Let $\cup_{i=1}^{i=k} \Gamma_{i}=\left\{z_{i}(-n)\right\}_{n \geq 0}$ be heteroclinic cycles connecting periodic points $x_{1}, x_{2}, \ldots, x_{k}$. By Definition 3, there exist constants $r_{i}>0, \lambda_{i}>1$ such that, for any $m \in\left[0, n_{i}-1\right] \cap \mathbb{N}, x, y \in \bar{B}_{r_{i}}\left(h^{m}\left(x_{i}\right)\right), \quad\|h(x)-h(y)\| \geq$ $\lambda_{i}\|x-y\|$. By Lemma 3 , for every $i \in[1, k] \cap \mathbb{N}$, there exists a positive constant $\lambda_{i 1}$, such that for any $g \in \mathscr{V}\left(h, \lambda_{i 1}\right)$ and $m \in\left[0, n_{i}-1\right] \cap \mathbb{N}$,

$$
m \in\left[0, n_{i}-1\right] \cap \mathbb{N},
$$

and $g$ is expanding on $B_{r_{i}}\left(g^{m}\left(x_{i}\right)\right)$. Since $\cup_{i=1}^{i=k} \Gamma_{i}=\left\{z_{i}(-n)\right\}_{n \geq 0}$ are heteroclinic cycles that connect periodic points $x_{1}, x_{2}, \ldots, x_{k}$, for any integer $i \in[1, k]$, there exists a positive integer $m_{i}$ such that $z_{i}\left(-m_{i}\right) \in B_{r_{i}}\left(x_{i}\right)$. Note that for any point $z \in \Gamma_{i}, D h(z)$ is an invertible operator. Therefore, by Lemma 2 , for every $m \in\left[0, m_{i}-1\right] \cap \mathbb{N}$, there exist an open neighborhood $V_{i m}$ of $h^{m}\left(z_{i}\left(-m_{i}\right)\right)$ and a positive constant $\lambda_{i 2}$ such that, for any $g \in \mathscr{V}\left(h, \lambda_{i 2}\right)$, $g: V_{i m} \longrightarrow V_{i(m+1)}$ is a differentiable homeomorphism. Without loss of generality, suppose that $V_{i 0} \subset B_{r_{i}}\left(x_{i}\right)$. Thus, $g$ is expanding in $V_{i 0}$.

Let $\lambda=\min \left\{\lambda_{i 1}, \lambda_{i 2} \mid 1 \leq i \leq k\right\}$. It is needed to prove that there exists a positive constant $\lambda_{0} \in(0, \lambda)$ such that, for any $g \in \mathscr{V}\left(h, \lambda_{0}\right), \quad g$ has heteroclinic cycles connecting expanding periodic points. Take $0<\widehat{r}_{i}<r_{i}$ with $B_{\widehat{r}_{i}}\left(x_{i}\right) \cap V_{i 0}=\varnothing . \quad$ Define a map

$H: \mathscr{V}(h, \lambda) \times V_{k 0} \times B_{\widehat{r}_{1}}\left(x_{1}\right) \times V_{10} \times B_{\widehat{r}_{2}}\left(x_{2}\right) \times V_{20} \times \cdots \times$ $V_{(k-1) 0} \times B_{\widehat{r}_{k}}\left(x_{k}\right) \longrightarrow \overbrace{\mathbb{R}^{n} \times \cdots \times \mathbb{R}^{n}}^{2 k}$ as

$$
\begin{aligned}
H\left(g, v_{k}, u_{1}, v_{1}, \ldots, u_{k}\right)= & \left(g^{m_{k}}\left(v_{k}\right)-u_{1}, g^{n_{1}}\left(u_{1}\right)\right. \\
& -u_{1}, g^{m_{1}}\left(v_{1}\right)-u_{2}, g^{n_{1}}\left(u_{2}\right) \\
& \left.-u_{2}, \ldots, g^{n_{k}}\left(u_{k}\right)-u_{k}\right) .
\end{aligned}
$$

Since $h^{n_{i}}$ is expanding at $x_{i}$, all eigenvalues of $D h^{n_{i}}\left(x_{i}\right)$ are greater than one in absolute value. Therefore, $D h^{n_{i}}\left(x_{i}\right)-$ $I$ is invertible. Note that $D h^{m_{i}}\left(z_{i}\left(-m_{i}\right)\right)$ is invertible. Therefore, the diagonal block linear operator

$$
\begin{aligned}
D_{z} H\left(h, z_{0}\right) & =\left(\left.\frac{\partial H(g, z)}{\partial z}\right|_{g=h, z_{0}=\left(z_{k}\left(-m_{k}\right), x_{1}, z_{1}\left(-m_{1}\right), \ldots, x_{k}\right)}\right) \\
& =\left[\begin{array}{cccccc}
D h^{m_{k}}\left(z_{k}\left(-m_{k}\right)\right) & -I & 0 & 0 & \cdots & 0 \\
0 & D h^{n_{1}}\left(x_{1}\right)-I & 0 & 0 & \cdots & 0 \\
\vdots & \vdots & \vdots & \vdots & \cdots & \vdots \\
0 & 0 & 0 & 0 & \cdots & D h^{n_{k}}\left(x_{k}\right)-I
\end{array}\right],
\end{aligned}
$$

is also invertible. Here we write

$$
\begin{aligned}
z= & \left(v_{k}, u_{1}, v_{1}, \ldots, u_{k}\right) \in V_{k 0} \times B_{\widehat{r}_{1}}\left(x_{1}\right) \times V_{10} \\
& \times B_{\widehat{r}_{2}}\left(x_{2}\right) \times V_{20} \times \cdots \times V_{(k-1) 0} \times B_{\widehat{r}_{k}}\left(x_{k}\right) .
\end{aligned}
$$

$H\left(h, z_{k}\left(-m_{k}\right), x_{1}, z_{1}\left(-m_{1}\right), \ldots, x_{k}\right)=0$, and if $i \neq j$, then $h^{m}\left(x_{i}\right) \neq x_{j}$ for any integer $m \in\left[1, n_{i}\right]$. By Lemma 1 , there exist constants $0<\lambda_{0}<\lambda, 0<r_{i 0}<\widehat{r}_{i}, 0<\delta_{i}$, and $\varphi: \mathscr{V}\left(h, \lambda_{0}\right) \longrightarrow B_{\delta_{k}}\left(z_{k}\left(-m_{k}\right)\right) \times B_{r_{10}}\left(x_{1}\right) \times B_{\delta_{1}}\left(z_{1}\left(-m_{1}\right)\right) \times$ $\cdots \times B_{r_{k 0}}\left(x_{k}\right)$ such that 
(1) $B_{\delta_{\dot{h}_{i}}}\left(z_{i}\left(-m_{i}\right)\right) \subset V_{i 0}, B_{\delta_{i}}\left(z_{i}\left(-m_{i}\right)\right) \cap B_{r_{i 0}}\left(x_{i}\right)=\varnothing$, $\cup_{m=1}^{i_{i}} h^{m}\left(B_{r_{i 0}}\left(x_{i}\right)\right) \cap B_{r_{j 0}}\left(x_{j}\right)=\varnothing$.

(2) For every $g \in \mathscr{V}\left(h, \lambda_{0}\right), \quad \varphi(g) \triangleq\left(\varphi_{1}(g)\right.$, $\left.\varphi_{2}(g), \ldots, \varphi_{2 k}(g)\right)$ is the unique solution to equations $g^{m_{k}}\left(v_{k}\right)=u_{1}, g^{n_{1}}\left(u_{1}\right)=u_{1}, \quad g^{m_{1}}\left(v_{1}\right)=u_{2}$, $g^{n_{2}}\left(u_{2}\right)=u_{2}, \ldots, g^{m_{k-1}}\left(v_{k-1}\right)=u_{k}$, and $g^{n_{k}}\left(u_{k}\right)=$ $u_{k}$, that is, $g^{m_{k}}\left(\varphi_{1}(g)\right)=\varphi_{2}(g), g^{n_{1}}$ $\left(\varphi_{2}(g)\right)=\varphi_{2}(g), \ldots, g^{n_{k}}\left(\varphi_{2 k}(g)\right)=\varphi_{2 k}(g)$.

Therefore, for any integer $j \in[1, k], \varphi_{2 j}(g)$ is expanding periodic point of $g$, and if $i \neq j, g^{m}\left(\varphi_{2 i}\right) \neq \varphi_{2 j}$ for any $m \in\left[1, n_{i}\right] \cap \mathbb{N}$. In particular, $\varphi(h)=\left(z_{k}\left(-m_{k}\right)\right.$, $\left.x_{1}, z_{1}\left(-m_{1}\right), \ldots, x_{k}\right)$.

It is needed to show that for every $j \in[1, k] \cap \mathbb{N}, g$ has a forward orbit $\Gamma_{j}$ of $\varphi_{2(t(j)+1)}(g)$ connecting periodic points $\varphi_{2 j}(g)$ and $\varphi_{2(t(j)+1)}(g)$. First, for every $g \in \mathscr{V}(h$, $\left.\lambda_{0}\right) \subset \cap_{j=1}^{k} \mathscr{V}\left(h, \lambda_{j 1}\right)$, there is

$$
B_{r_{j}}\left(x_{j}\right) \subset g^{n_{j}}\left(B_{r_{j}}\left(x_{j}\right)\right)
$$

and $g^{n_{j}}$ is expanding on $B_{r_{j}}\left(x_{j}\right)$. Let $\left(\widetilde{g}^{n_{j}}\right)^{-1}=\left(\left.g^{n_{j}}\right|_{B_{r_{j}}\left(x_{j}\right)}\right)^{-1}$ denote the inverse of $g^{n_{j}}$ restricted to $B_{r_{j}}\left(x_{j}\right)$, and the $l$-th iterate of $\left(\widetilde{g}^{n_{j}}\right)^{-1}$ denotes $\left(\widetilde{g}^{n_{j}}\right)^{-1}$. For every integer $l \geq 1$, let $\widehat{z}_{j}\left(-m_{j}\right)=\varphi_{2(t(j)+1)}(g), \widehat{z}_{j}\left(-\ln _{j}-m_{j}\right)=\left(\widetilde{g}^{n_{j}}\right)^{-l}\left(\widehat{z}_{j}\left(-m_{j}\right)\right)$. For any $x, y \in B_{r_{j}}\left(x_{j}\right)$, because

$$
\left\|\left(\tilde{g}^{n_{j}}\right)^{-1}(x)-\left(\tilde{g}^{n_{j}}\right)^{-1}(y)\right\|<\hat{\lambda}_{j}^{-1}\|x-y\|, \quad \hat{\lambda}_{j}>1,
$$

there is

$$
\begin{aligned}
\| \widehat{z}_{j} & \left(-\ln _{j}-m_{j}\right)-\varphi_{2 j}(g) \| \\
= & \left\|\left(\tilde{g}^{n_{j}}\right)^{-l}\left(\widehat{z}_{j}\left(-m_{j}\right)\right)-\left(\tilde{g}^{n_{j}}\right)^{-l}\left(\varphi_{2 j}(g)\right)\right\| \\
& <\hat{\lambda}_{j}^{-l}\left\|\varphi_{2(t(j)+1)}(g)-\varphi_{2 j}(g)\right\| \leq \hat{\lambda}_{j}^{-l} r_{j} .
\end{aligned}
$$

This proves that $\lim _{l \longrightarrow \infty} \widehat{z}_{j}\left(-l m_{j}\right)=\varphi_{2 j}(g)$.

For any integer $l \geq 1$ and $n \in\left((l-1) n_{j}, \ln _{j}\right) \cap \mathbb{N}$, let

$$
\widehat{z}_{j}\left(-n-m_{j}\right)=g^{\ln _{j}-n}\left(\widehat{z}_{j}\left(-\ln _{j}-m_{j}\right)\right),
$$

and for any integer $n \in\left[0, m_{j}\right]$, let

$$
\widehat{z}_{j}(-n)=g^{m_{j}-n}\left(\widehat{z}_{j}\left(-m_{j}\right)\right) .
$$

Therefore, $\left\{\widehat{z}_{j}(-n)\right\}_{n \in \mathbb{N}}$ is a forward orbit of $\varphi_{2(t(j)+1)}(g)$ connecting $\varphi_{2 j}(g)$ and $\varphi_{2(t(j)+1)}(g)$.

Because for every $0 \leq m \leq m_{i}-1, g: V_{i m} \longrightarrow V_{i(m+1)}$ is a differentiable homeomorphism, $D g^{m_{i}}\left(\widehat{z}_{j}\left(-m_{j}\right)\right)$ is an invertible linear operator. Moreover, for any $g \in \mathscr{V}\left(h, \lambda_{0}\right) \subset \mathscr{V}\left(h, \lambda_{j 1}\right), B_{\delta_{j}}\left(\widehat{z}_{j}\left(-m_{j}\right)\right) \subset V_{j 0} \subset B_{r_{j}}\left(x_{j}\right)$, so $\tilde{g}^{-\ln _{j}}\left(\widehat{z}_{j}\left(-m_{j}\right)\right) \in B_{r_{j}}\left(x_{j}\right)$ for $l \geq 1$. This implies that $D g^{n_{j}}\left(g^{-\ln _{j}}\left(\widehat{z}_{j}\left(-m_{j}\right)\right)\right.$ is an invertible linear operator for any integer $l \geq 1$. This proves that $\left\{\widehat{z}_{j}(-n)\right\}_{n \in \mathbb{N}}$ satisfies condition (3) in Definition 3. Let $\Gamma_{j}=\left\{\widehat{z}_{j}(-n)\right\}_{n \geq 0}$ for any integer $j \in[1, k]$. Therefore, $\cup_{j=1}^{k} \Gamma_{j}$ are heteroclinic cycles connecting expanding periodic points $\varphi_{2}(g), \varphi_{4}(g), \ldots, \varphi_{2 k}(g)$.

\section{Examples}

In the following, two examples are given which illustrate our results and applications.

Example 1. Consider the one-dimensional map

$$
f(x)= \begin{cases}-4(x(1-x)), & x \in[0,1] \\ -4(x(1+x)), & x \in[-1,0)\end{cases}
$$

$f$ has only one fixed point 0 , and thus $f$ does not have heteroclinic repellers. Directing calculation, we have

(1) $f$ has three 2-periodic points which are not in the same orbit $\quad x_{1} \approx 0.3454915, x_{2}=0.75$, $x_{3} \approx 0.9045084$.

(2) $f^{2}$ is expanding in $(0.16325,0.4684)$, $(0.5315,0.8367),(0.8682,1)$.

(3) $f^{2}(0.25)=0.75, f^{2}(0.6545085)=0.3454915$.

Therefore, $f$ has heteroclinic cycles connecting expanding periodic points $x_{1} \approx 0.3454915$ and $x_{2}=0.75$ (see Figure 1).

Remark 2. Example 1 shows that $f$ does not have heteroclinic repellers but $f$ has heteroclinic cycles connecting expanding periodic points. So, heteroclinic cycles connecting expanding periodic points really contain heteroclinic repellers.

Example 2. Recall the wave equation with a van der Pol boundary condition [3], as follows:

$$
\begin{cases}w_{t t}-w_{x x}=0, & x \in(0,1), t>0, \\ w_{x}(0, t)=-\eta w_{t}(0, t), & \eta \neq 1, \\ w_{x}(1, t)=\alpha w_{t}(1, t)-\beta w_{t}^{3}(1, t), & 0<\alpha<1, \beta>0, t>0, \\ w(x, 0)=w_{0}(x), w_{t}(x, 0)=w_{1}(x), & 0<x<1 .\end{cases}
$$

Let

$$
\begin{aligned}
& G_{\eta}(x)=\frac{\eta+1}{\eta-1} x, \quad \eta \neq 1, \\
& F_{\alpha}(x)=p(x)+x,
\end{aligned}
$$

where $y=p(x)$ is the unique real solution of the cubic equation

$$
\beta y^{3}+(1-\alpha) y+2 x=0
$$

Let $\quad u(x, t)=\left(\left(w_{x}(x, t)+w_{t}(x, t)\right) /\right.$ 2), $v(x, t)=\left(\left(w_{x}(x, t)-w_{t}(x, t)\right) / 2\right)$. Then, the solution $(u, v)$ of system $(30)$ is completely characterized by the interval maps $G_{\eta} \circ F_{\alpha}(\cdot)$ and $F_{\alpha} \circ G_{\eta}(\cdot)$, between which there is topological conjugacy. If $G_{\eta} \circ F_{\alpha}(\cdot)$ is chaotic on some invariant interval, we say that the gradient $w$ of system (30) is chaotic. Chen. et al. have analyzed the chaos caused by snapback repellers in system (30) (see [3]). For example, when $\alpha=0.6, \beta=1$ and $\eta=0.82,\left(G_{\eta} \circ F_{\alpha}\right)^{4}(\cdot)$ has a snapback repeller. It can be checked that $G_{\eta} \circ F_{\alpha}(\cdot)$ has 


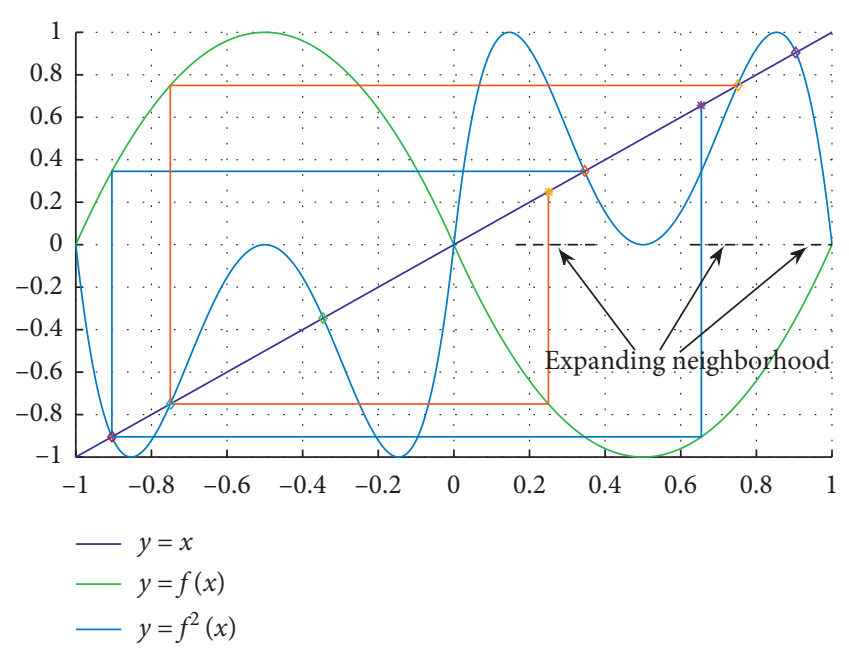

FIgURE 1: Heteroclinic cycles connecting expanding periodic points.

heteroclinic cycles connecting expanding periodic points. In particular, it is easier to find the conditions that $G_{\eta} \circ F_{\alpha}(\cdot)$ has heteroclinic cycles.

\section{Conclusions}

In this paper, a new criterion of chaos is established in $\mathbb{R}^{n}$. By constructing shift invariant sets, we prove that heteroclinic cycles imply chaos in the sense of Devaney. Heteroclinic cycles connecting expanding periodic points is $C^{1}$ structural stability.

\section{Data Availability}

No data were used to support this study.

\section{Conflicts of Interest}

The author declares that there are no conflicts of interest regarding the publication of this paper.

\section{Acknowledgments}

This study was supported in part by the National Natural Science Foundation of China (11671410 and 61907010), the Natural Science Foundation of Guangdong Province (2018A0303130120), and the Foundation for Natural Science in Higher Education of Guangdong, China (2019KZDXM036).

\section{References}

[1] T.-Y. Li and J. A. Yorke, "Period three implies chaos," The American Mathematical Monthly, vol. 82, no. 10, pp. 985-992, 1975.

[2] G. C. Blanco, "Chaos and topological entropy in dimension $n>1$," Ergodic Theory Dynamical Systems, vol. 6, pp. 163-165, 1986.

[3] G. Chen, S.-B. Hsu, and J. Zhou, "Snapback repellers as a cause of chaotic vibration of the wave equation with a van der Pol boundary condition and energy injection at the middle of the span," Journal of Mathematical Physics, vol. 39, no. 12, pp. 6459-6489, 1998.

[4] Y. Chen, L. Li, X. Wu, and F. Wang, "The structural stability of maps with heteroclinic repellers," International Journal of
Bifurcation and Chaos, vol. 30, no. 14, p. 10, Article ID 2050207, 2020.

[5] Y. Chen and X. Wu, "Chaos on discrete neural network loops with self-feedback," Discrete Dynamics in Nature and Society, vol. 2020, pp. 1-9, Article ID 3528684, 2020.

[6] X. Wu, Y. Chen, and F. Wang, "Chaotic criteria in complete metric spaces(in Chinese)," Acta Mathematica Sinica Chinese Series, vol. 64, no. 2, pp. 225-230, 2021.

[7] Y. Chen and S. Luo, "The Lipschitz perturbations of regular nondegenrate heteroclinic cycles in Banach spaces(in Chinese)," Acta Mathematica Sinica Chinese Series, vol. 64, no. 3, pp. 1-7, 2021.

[8] M. Saleh and J. Ma, "Robust finite-time composite nonlinear feedback control for synchronization of uncertain chaotic systems with nonlinearity and time-delay," Chaos Solitons \& Fractals, vol. 114, pp. 46-54, 2018.

[9] M. Saleh, V. Sundarapandian, S. Aceng et al., "A novel chaotic system with boomerang-shaped equilibrium, its circuit implementation and application to sound encryption," Iranian Journal of Science and Technology, Transactions of Electrical Engineering, vol. 43, pp. 1-12, 2018.

[10] X. Wu, Y. Chen, L. Liang, and F. Wang, "Complex dynamics of discrete-time ring neural networks," International Journal of Bifurcation and Chaos, vol. 31, no. 8, p. 12, Article ID 2150116, 2021

[11] F. R. Marotto, "Snap-back repellers imply chaos in $R n$," Journal of Mathematical Analysis and Applications, vol. 63, no. 1, pp. 199-223, 1978.

[12] R. L. Devaney, An Introduction to Chaotic Dynamical Systems, Addison-Wesley Publishing Company, Redwood City, CA, USA, Second edition, 1989.

[13] W. Huang and X. Ye, "Devaney's chaos or 2-scattering implies Li-Yorke's chaos," Topology and Its Applications, vol. 117, no. 3, pp. 259-272, 2002.

[14] Y. Shi and G. Chen, "Chaos of discrete dynamical systems in complete metric spaces," Chaos, Solitons \& Fractals, vol. 22, no. 3, pp. 555-571, 2004.

[15] Y. Shi and G. R. Chen, "Discrete chaos in banach spaces," Science in China Series A, vol. 48, no. 2, pp. 222-238, 2005.

[16] Y. Chen and $\mathrm{X}$. $\mathrm{Wu}$, "The $C 1$ persistence of heteroclinic repellers in Rn," Journal of Mathematical Analysis and Applications, vol. 485, no. 2, Article ID 123823, 2020.

[17] W. Lin and G. Chen, "Heteroclinical repellers imply chaos," International Journal of Bifurcation and Chaos, vol. 16, no. 05, pp. 1471-1489, 2006.

[18] Z. Li, Y. Shi, and W. Liang, "Discrete chaos induced by heteroclinic cycles connecting repellers in banach spaces," Nonlinear Analysis: Theory, Methods \& Applications, vol. 72, no. 2, pp. 757-770, 2010.

[19] F. R. Marotto, "Chaotic behavior in the Henon mapping," Communications in Mathematical Physics, vol. 68, no. 2, pp. 187-194, 1979.

[20] M.-C. Li and M.-J. Lyu, "A simple proof for persistence of snap-back repellers," Journal of Mathematical Analysis and Applications, vol. 352, no. 2, pp. 669-671, 2009.

[21] Y. Chen, Y. Huang, and L. Li, "The persistence of snap-back repeller under small C1 perturbations in banach spaces," International Journal of Bifurcation and Chaos, vol. 21, no. 03, pp. 703-710, 2011.

[22] H.-J. Chen and M.-C. Li, "Stability of symbolic embeddings for difference equations and their multidimensional perturbations," Journal of Differential Equations, vol. 258, no. 3, pp. 906-918, 2015. 